\title{
Post-operative infection in mechanical circulatory support patients
}

\author{
Zihui Tan, Ling Antonia Zeng \\ Department of Anaesthesiology, Division of Anaesthesiology and Perioperative Medicine, Singapore General Hospital, Singapore \\ Contributions: (I) Conception and design: Z Tan; (II) Administrative support: Z Tan; (III) Provision of study materials or patients: All authors; (IV) \\ Collection and assembly of data: All authors; (V) Data analysis and interpretation: All authors; (VI) Manuscript writing: All authors; (VII) Final \\ approval of manuscript: All authors. \\ Correspondence to: Zihui Tan. Associate Consultant, Department of Anesthesia, Singapore General Hospital, Outram Road, Singapore. \\ Email: tan.zihui@singhealth.com.sg.
}

\begin{abstract}
The management of end stage heart failure has changed dramatically in recent years with the advent of mechanical circulatory support devices as well as rapid improvement and increased availability of these devices. With the improvements in survival and quality of life in these patients, post-operative infections become a significant contribution to morbidity and mortality. Post-operative infections need to be adequately addressed in a timely fashion by early diagnosis and appropriate treatment. Recognizing risk factors for infection and instituting good infection control measures is also key in caring for these patients. Multiple patient and device factors have been shown to be correlated with increased post-operative infections, and cellular immunity is also impaired in patients on ventricular assist devices (VAD). Cultures should be taken prior to starting antimicrobial treatment. Empirical treatment needs to account for common pathogens, local microbial resistance and subsequently be culture guided once results are available. Patients on extracorporeal membrane oxygenation present a unique challenge with drug dosing due to altered pharmacokinetics. VAD related and VAD specific infections require appropriate wound care and possible surgical intervention. This narrative review summarizes the literature available for the management and prevention of post-operative infections in patients with mechanical circulatory devices. Vigilance in identifying risk factors, prompt treatment and active prevention is crucial to the management.
\end{abstract}

Keywords: Infection; extracorporeal membrane oxygenation (ECMO); mechanical circulatory support (MCS); ventricular assist devices (VAD)

Submitted Feb 21, 2020. Accepted for publication May 13, 2020.

doi: 10.21037/atm-20-1748

View this article at: http://dx.doi.org/10.21037/atm-20-1748

\section{Introduction}

Mechanical circulatory support devices such as left ventricular assist devices (LVAD) are increasingly important in the management of end stage heart failure. Newer and better devices have contributed to improvements in mortality as well as quality of life but post-operative infections occur in up to $60 \%$ of this group of patients (1). This narrative review aims to discuss the types of infection as well as the risk factors, treatment and prevention of associated infections.

\section{Types of devices}

Devices used for mechanical circulatory support (MCS) can be divided into short and long-term devices, these devices provide circulatory support by performing work for a failing left or right ventricle or both.

Short-term devices are designed to provide hemodynamic support for a wide range of clinical conditions, and are used for durations ranging from days to weeks. The most used device world-wide is the intra-aortic balloon pump and consists of a counter-pulsation pump placed percutaneously 
in the descending aorta (2).

Other devices include the Impella (Abiomed, Danvers, MA, USA), a continuous non-pulsatile axial flow Archimedes-screw pump that expels aspirated blood from left ventricle into ascending aorta (3). The Tandemheart (TandemLife, Pittsburgh PA, USA) is a percutaneous continuous flow centrifugal pump providing support of up to $4 \mathrm{~L} / \mathrm{min}$; the inflow cannula is placed via trans-septal puncture into left atrium and outflow in lower abdominal aorta (4). Similar to IABP, there is no evidence to suggest that they improve clinical outcome (4-7).

Veno-arterial extracorporeal membrane oxygenation (V-A ECMO) device is a full cardiopulmonary support system similar to the heart-lung bypass machine used in cardiac surgery. It involves placement of large bore catheters that allow drainage of deoxygenated blood via a venous cannula from the right atrium, the blood passes through an oxygenator where gas exchange occurs (carbon dioxide removal and oxygenation) and oxygenated blood returns via an arterial cannula to the aorta using a centrifugal pump, thus bypassing the pulmonary circulation. VA-ECMO offers extended support to patients in cardiac and/or respiratory failure and is considered a viable lifesaving modality (8).

Technological advancement has improved durable MCS devices over the last decade, substantial progress has been made in durability, size, biocompatibility and reliability. Currently, ventricular assist devices (VAD) and total artificial hearts (TAHs) are used as a bridge to transplant. A VAD is used also as bridge to wait listing (candidacy for heart transplant), but may just as well be explanted in the rare case of cardiac recovery. Patients who are not a transplant candidate may qualify for destination therapy, i.e., a VAD is implanted for permanent, life-long support.

The first generation VAD devices were large pulsatile devices with a paracorporeal pump, or an intracorporeal pump placed in the abdomen or pre-peritoneum with a driveline coming out through the skin. Second generation devices include the continuous flow rotary pump, most common the HeartMate II (Abbott, St. Paul, MN, USA) that significantly benefitted patients with end stage heart failure (9). The third generation VADs are continuous flow devices with a centrifugal pump design placed in the pericardial space, notably the HeartWare (Medtronic, Minneapolis, MN, USA) (10) and the latest device HeartMate III with a fully magnetically levitated impeller (11).

Biventricular MCS outcomes are less encouraging (12). More patients are receiving 2 continuous flow pumps for biventricular support as off-label use-primarily the
HVAD (13), but also the HeartMate III (14) and even the Jarvik 2000 (15), have been used clinically as an alternative to extracorporeal biventricular assist devices (BIVAD) or TAH. The TAH consists of 2 intracorporeal, pneumatic replacement pumps with transcutaneous air power tubes connected to a computer-assisted driving unit. The Syncardia TAH (Tucson, AZ, USA) has substantially reduced weight and size since the first device and is still in use today (16).

Clinically important BIVADs include the Thoratec (Pierce-Donachy) VA (Pleasanton, CA, USA) and the Berlin Heart Excor. Both are extracorporeal replacement pumps with transcutaneous guided cannulas for blood drainage and supply $(17,18)$.

\section{Risk factors}

\section{Type of device}

The continuous-flow devices are smaller, with reduced surface areas of foreign material, less pump movement inside the body and less surgical dissection to implant with lower device-related infection rates $(19,20)$. The fifth INTERMACS annual report, with risk factor analysis of more than 6,000 patients, the rate (events/100 patient months) of infection was 22.81 and $8.01(\mathrm{P}<0.0001)$ in pulsatile and continuous-flow devices, respectively (21). Xie et al. in an IMACS analysis of more than 15,000 patients from 2013-2017, found that axial pumps had a higher rate of developing VAD infections compared to centrifugal pumps, notably younger patients had a higher hazard of VAD infection in this group (22).

\section{Duration of support}

Infection rates increase with duration of MCS support, especially for driveline infections for VADs (19,23). A single centre Italian study found that patients with infection had longer ECMO support duration, VAD support duration, intensive care unit stay and hospital stay than patients without infection (24).

\section{Host factors}

Higher body mass index has been shown to independently correlate with higher rates of both device related and non-VAD related infections (25), including blood stream infections (26). 


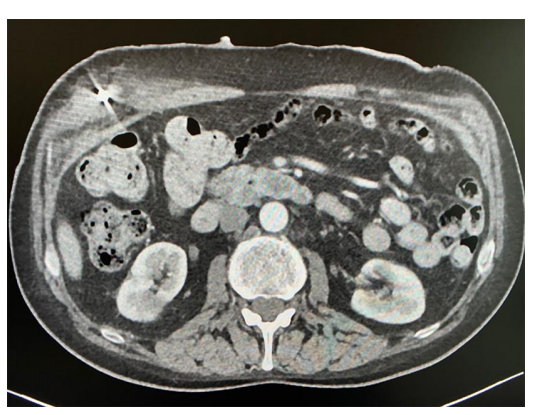

Figure $1 \mathrm{CT}$ scan showing driveline infection in the right upper abdominal area.

Other patient factors that have been reported include diabetes mellitus (27), older age (28), chronic renal disease and dialysis $(29,30)$, pre-implant frailty and Interagency Registry for Mechanically Assisted Circulatory Support category 1 (26).

Poston and colleagues reported hypoalbuminaemia (as a predictor of poor nutritional status) predicted device related bloodstream infections (31). A high bilirubin as an indicator of right sided heart failure has also been found to correlate with device related blood stream infection (26). Postoperative bleeding necessitating re-operation is an important contributing factor for infectious complications in MCS (32).

Cellular immunity is impaired among patients on left ventricular assist device because of a downregulatory cytokine imbalance and emergence of suppressive T-regulatory cells (33). VAD patients with hypogammaglobulinemia (defined as immunoglobulin $\mathrm{G}<700 \mathrm{mg} / \mathrm{dL}$ ) are at increased risk of infection (34).

\section{Types of infection-ventricular assist devices}

The International Society of Heart and Lung Transplantation (ISHLT) consensus document for prevention and management strategies for mechanical circulatory support infection divided infections into three categories: VAD specific, VAD related and non VAD infections (1). VAD specific commonly include driveline infection, pocket infection as well as pump or cannula infection. These can be complicated by systemic blood stream infections, infective endocarditis and mediastinitis (VAD related) which can cause high morbidity and mortality. Other infections not specific to VAD patients include hospital acquired pneumonia, urinary tract infection, line or blood stream infections which can occur in any critically ill patient in the hospital setting.

Driveline infections are the most common of the VAD specific infections (35). Driveline infection can present as erythema, tenderness as well as purulent discharge of the entry site of the device. They can be superficial or track deep into the subcutaneous tissue (Figure 1) (36). Diagnosis can be confirmed by a wound swab culture or tissue culture after debridement. Ultrasound can be used to detect fluid pockets but computed tomography (CT) has limited benefit due to the artefact caused by the device. A case series has explored the use of gallium single photon emission tomography-CT (SPECT-CT) for extent of infection but this has not been widely adopted yet (37).

Pocket Infection is the second most common VAD specific infection (35). This pocket is created in the preperitoneal space to house the pump. Haematoma and fluid can collect and organisms can grow in this nonvascular space. Infection may also arise from an extension of the driveline infection $(29,38)$.

Driveline infections are often difficult to treat due to the permanent nature of the device. Relapses occur and resistance may develop. The main pathogens are Gram positive bacteria especially Staphylococcus aureus and Staphylococcus epidermidis causing more than $50 \%$ of MCS infections $(29,39)$. The most common Gram negative bacteria is Pseudomonas aeruginosa followed by Enterobacter (1). Fungal infections are rare but results in high mortality $(40,41)$. Candida albicans is the most common (70\%) followed by Candida glabrata (10\%) (1).

Blood stream infections are found to be associated with increased risk of haemorrhagic and ischaemic stroke and longer hospitalisation (35).

\section{Treatment}

Wound swab and blood and fluid cultures should be taken before starting broad spectrum antibiotics. Treatment of driveline infections should be empirical initially to cover the organisms mentioned above including MRSA. Antibiotic choice should take into consideration the institution's microbial resistance history, the patient's previous culture results as well as antimicrobial history (29). Culture directed antimicrobial therapy can then be instituted when results are available.

Proper wound care is also essential in the treatment. Surgical debridement is reserved for deep infections (42). Application of a VAC system can be considered in deep infections after surgical drainage. Device exchange is a last 
resort as it involves high morbidity and mortality (1).

There is no recommendation for duration of antimicrobial therapy. However, it is reasonable to continue until patient is stable after device exchange or heart transplant. A protracted course of antimicrobial therapy may be offered when there is evidence in preoperative or intraoperative positive cultures (1).

\section{Prevention}

Prior to instituting mechanical circulatory support, all patients should be screened for active infections. Infectious disease consult should be performed in patients with suspected or confirmed infection. ISHLT also published a series of recommendations for the care of invasive lines in these patients which include: avoiding femoral lines, tunneled catheters for prolonged use as well as avoiding exchange over a wire. Lines should be examined daily and removed if there is evidence of infection (1).

With regards to intraoperative care, apart from standard infection control measures, pre-operative prophylaxis is recommended and should be given within $60 \mathrm{~min}$ of first incision and not continued beyond 24-48 hours (42). Vancomycin infusion should be started within 2 hours. Although there is no specific choice of antimicrobial, it should provide good Staphylococcus spp coverage as well as MRSA if there is a high prevalence in the centre. Routine use of antifungal and gram-negative coverage is not necessary. Antibiotics may be re-dosed in surgery longer than 2 half-lives of the drug or if there is significant blood loss (>1,500 $\mathrm{mL}$ or 2 units of packed red cells given).

Other intraoperative measures include good glycaemic control, transfusion as necessary and not withheld during and in the immediate post-operative period as well as securing the drive line exit site so as not to cause shearing are recommended (1).

Post operatively, nursing care of the drive line should be performed under sterile conditions (43). Patients and caregivers should be trained in the care of the MCS. The drive line should be stabilized with a binder or anchoring device and infection surveillance must be performed frequently (1).

\section{Extracorporeal membrane oxygenation}

In the adult population, blood stream infections range from $3-18 \%$ and the incidence is reported to be 2.98 to
20.55 episodes per 1,000 ECMO days. Lower respiratory tract infection rates are also high at 24.4 episodes per 1,000 ECMO days. Hospital acquired infections increase mortality risk by 38-63\% (24).

There is also a recent study looking specifically at ECMO device related infection. Thomas et al. found an incidence of $9.7 \%$ in $105 \mathrm{VV}$-ECMO patients as well as an ECMO device colonization rate of $32 \%$. In his study, ECMO device related infections involves the drainage cannula, return cannula as well as the membrane oxygenator. This group of patients not surprisingly have a longer ECMO duration (44).

Infections not only increase length of hospital stay and duration of ventilatory support (45). It can cause problems within the circuit since it activates the coagulation systems and thus can result in clot formation (24).

For blood stream infections, coagulase negative staphylococcus is the most common pathogen, followed by Candida spp and Pseudomonas aeruginosa. With regards to ventilator associated infections, Enterobacteriaceae has been reported as the most common pathogen, accounting for $31 \%$ of all reported cases $(24,46)$.

Over the years, there have been many studies looking at risk factors for infection during extracorporeal membrane use. O'Neill et al. found that presence of an open chest and procedures on ECMO are associated with a higher risk of infection (47). Other studies found that duration of ECMO (48-50), severity of the disease (24), mechanical complications, autoimmune disease and venovenous mode (48) are associated with a higher risk of infection.

\section{Treatment}

Early focused antimicrobial therapy is important to prevent severe infection complications (51). The challenge of treatment of infections in ECMO patients lie in the altered pharmacokinetics of the antimicrobial therapy. Most of the pharmacokinetics studies so far have been conducted with gentamicin and vancomycin and mainly in the neonatal population. There is a larger volume of distribution due to the increased circuit volume and decreased clearance for both drugs $(52,53)$. The reasons for the decreased clearance are multifactorial although the primary reason is decreased renal function (52). Other factors that affect drug delivery include losses during equipment change as well as sequestration due to adhesion to circuit components. The addition of haemofiltration further complicates matters.

Lipophilic and highly protein bound drugs are 
significantly sequestered in the circuit while hydrophilic drugs like beta lactams and glycopeptides are more affected by haemodilution (54). However, the extent to which this occurs varies from drug to drug. The literature out there suggests individualising drug regimen through therapeutic drug monitoring $(55,56)$.

\section{Prevention}

The ELSO infectious disease task force has produced a list of recommendations for prevention of nosocomial infection. Apart from standard infection control such as frequent hand washing and use of sterile practices when handling lines, they also include reducing unnecessary interruption of lines during the care of the patient, needleless hub for all connections, avoiding unnecessary lines and long-term vascular access in these patients as well as use of chlorhexidine as antiseptic is advised. There must be careful isolation of patients infected with multi-drug resistant organisms. System infection prevention is also important and these include active prevention of ventilator associated pneumonia, an early shift to enteral nutrition and if parenteral nutrition is necessary, to be given through a dedicated access port (57).

Antibiotic prophylaxis remains highly controversial due to concerns of multi-drug resistant organisms as well as Clostridium difficile associated colitis (58). Biffi et al. found no data at present supporting the use of antibiotic prophylaxis to prevent infections during ECMO (24). Although an indication for antibiotic prophylaxis may exist in some subpopulations such as the patients with an open chest, current evidence is still poor (59).

\section{Conclusions}

Post-operative infection in MCS patients is a serious complication with significant morbidity and mortality. With the standardization of definitions in recent years as well as task force recommendations, improvement can be made in the management of post-operative infections as well as elucidate better preventive measures with further research.

\section{Acknowledgments}

The authors would like to acknowledge Associate Professor Thuan Tong Tan, Head and Senior Consultant, Department of Infectious Diseases, Singapore General Hospital for his contribution.

Funding: None.

\section{Footnote}

Provenance and Peer Review: This article was commissioned by the Guest Editor (Kamen Valchanov) for the series "Perioperative Management of Patients with undergoing Mechanical Circulatory Support" published in Annals of Translational Medicine. The article was sent for external peer review organized by the Guest Editor and the editorial office.

Conflicts of Interest: Both authors have completed the ICMJE uniform disclosure form (available at http:// dx.doi.org/10.21037/atm-20-1748). The series "Perioperative Management of Patients with undergoing Mechanical Circulatory Support" was commissioned by the editorial office without any funding or sponsorship. The authors have no other conflicts of interest to declare.

Ethical Statement: The authors are accountable for all aspects of the work in ensuring that questions related to the accuracy or integrity of any part of the work are appropriately investigated and resolved.

Open Access Statement: This is an Open Access article distributed in accordance with the Creative Commons Attribution-NonCommercial-NoDerivs 4.0 International License (CC BY-NC-ND 4.0), which permits the noncommercial replication and distribution of the article with the strict proviso that no changes or edits are made and the original work is properly cited (including links to both the formal publication through the relevant DOI and the license). See: https://creativecommons.org/licenses/by-nc-nd/4.0/.

\section{References}

1. Kusne S, Mooney M, Danziger-Isakov L, et al. An ISHLT consensus document for prevention and management strategies for mechanical circulatory support infection. J Heart Lung Transplant 2017;36:1137-53.

2. Kapelios CJ, Terrovitis JV, Nanas JN. Current and future applications of the intra-aortic balloon pump. Curr Opin Cardiol 2014;29:258-65. 
3. Ouweneel DM, Eriksen E, Seyfarth M, et al. Impella Versus Intra-Aortic Balloon Pump For Treatment Of Cardiogenic Shock: A Meta-Analysis of Randomized Controlled Trials. J Am Coll Cardiol 2017;69:358.

4. Mandawat A, Rao SV. Percutaneous Mechanical Circulatory Support Devices in Cardiogenic Shock. Circ Cardiovasc Interv 2017;10. doi: 10.1161/ CIRCINTERVENTIONS.116.004337.

5. Unverzagt $S$, Buerke $M$, de Waha A, et al. Intra-aortic balloon pump counterpulsation (IABP) for myocardial infarction complicated by cardiogenic shock. Cochrane Database Syst Rev 2015;(3):CD007398.

6. Schrage B, Ibrahim K, Loehn T. Impella Support for Acute Myocardial Infarction Complicated by Cardiogenic Shock : A Matched-Pair IABP-SHOCK II Trial 30-Day Mortality Analysis. Circulation 2019;140:e559-e560.

7. Thiele H, Jobs A, Ouweneel DM, et al. Percutaneous short-term active mechanical support devices in cardiogenic shock: a systematic review and collaborative meta-analysis of randomized trials. Eur Heart J 2017;38:3523.

8. Keebler ME, Haddad EV, Choi CW et al. Venoarterial Extracorporeal Membrane Oxygenation in Cardiogenic Shock. JACC Heart Fail 2018;6:503-16.

9. Miller LW, Pagani FD, Russell SD, et al. Use of a Continuous-Flow Device in Patients Awaiting Heart Transplantation. N Engl J Med 2007;357:885-96.

10. Wieselthaler GM, Driscoll GO, Jansz P, et al. Initial clinical experience with a novel left ventricular assist device with a magnetically levitated rotor in a multi-institutional trial. J Heart Lung Transplant 2010;29:1218-25.

11. Chatterjee A, Feldmann C, Hanke JS, et al. The momentum of HeartMate 3: a novel active magnetically levitated centrifugal left ventricular assist device (LVAD). J Thorac Dis 2018;10:S1790-3.

12. Kirklin JK, Naftel DC, Pagani FD, et al. Seventh INTERMACS annual report: 15,000 patients and counting. J Heart Lung Transplant 2015;34:1495-504.

13. Tran HA, Pollema TL, Silva Enciso J, et al. Durable Biventricular Support Using Right Atrial Placement of the HeartWare HVAD. ASAIO J 2018;64:323-7.

14. Potapov EV, Kukucka M, Falk V, et al. Biventricular support using 2 HeartMate 3 pumps. J. Heart Lung Transplant 2016;35:1268-70.

15. Brenner P, Wirth TJ, Liebermann A, et al. First Biventricular Jarvik 2000 Implants (Retroauricular Version) Via a Median Sternotomy. Exp Clin Transplant 2016;14:215-23.
16. Slepian MJ, Alemu Y, Girdhar G, et al. The Syncardia (TM) total artificial heart: in vivo, in vitro, and computational modeling studies. J Biomech 2013;46:266-75.

17. Latrémouille C, Carpentier A, Leprince P, et al. A bioprosthetic total artificial heart for end-stage heart failure: Results from a pilot study. J Heart Lung Transplant 2018;37:33-7.

18. Brehm K, Heilmann C, Siepe M, et al. Thoratec paracorporeal biventricular assist device therapy: the Freiburg experience. Eur J Cardiothorac Surg 2012;41:207-12.

19. Koval CE, Rakita R, AST Infectious Diseases Community of Practice. Ventricular assist device-related infections and solid organ transplantation. Am J Transplant 2013;13 Suppl 4:348-54.

20. Slaughter MS, Rogers JG, Milano CA, et al. Advanced Heart Failure Treated with ContinuousFlow Left Ventricular Assist Device. N Engl J Med 2009;361:2241-51.

21. Kirklin JK, Naftel DC, Kormos RL, et al. Fifth INTERMACS annual report : Risk factor analysis from more than 6,000 mechanical circulatory support patients. J Heart Lung Transplant 2013;32:141-56.

22. Xie R, Cowger J, Kirkland JK, et al. Epidemiology, Outcomes, and Effects of Device Flow Type on Ventricular Assist Devices (VAD) Infections: An IMACS Registry Analysis. J Heart Lung Transplant 2019;38:S101.

23. Zierer A, Melby SJ, Voeller RK, et al. Late-Onset Driveline Infections: The Achilles 'Heel of Prolonged Left Ventricular Assist Device Support. Ann Thorac Surg 2007;84:515-20.

24. Biffi S, Di S, Scaravilli V, et al. Infections during extracorporeal membrane oxygenation : epidemiology , risk factors, pathogenesis and prevention. Int J Antimicrob Agents 2017;50:9-16.

25. Forest SJ, Xie R, Kirklin JK, et al. Impact of body mass index on adverse events after implantation of left ventricular assist devices: An IMACS registry analysis. J Heart Lung Transplant 2018;37:1207-17.

26. Aslam S, Xie R, Cowger J, et al. Bloodstream infections in mechanical circulatory support device recipients in the International Society of Heart and Lung Transplantation Mechanically Assisted Circulation Support Registry: Epidemiology, risk factors, and mortality. J Heart Lung Transplant 2018;37:1013-20.

27. Martin SI. Infectious Complications of Mechanical Circulatory Support ( MCS ) Devices. Curr Infect Dis Rep 
2013;15:472-7.

28. Herrmann M, Weyand M, Greshake B, et al. Left Ventricular Assist Device Infection Is Associated With Increased Mortality but Is Not a Contraindication to Transplantation. Circulation 1997;95:814-7.

29. Gordon RJ, Quagliarello B, Lowy FD. Ventricular assist device-related infections. Lancet Infect Dis 2006;6:426-37.

30. Holman WL, Park SJ, Long JW, et al. Infection in permanent circulatory support: Experience from the REMATCH trial. J Heart Lung Transplant 2004;23:1359-65.

31. Poston RS, Husain S, Sorce D, et al. LVAD bloodstream infections: therapeutic rationale for transplantation after LVAD infection. J Heart Lung Transplant 2003;22:914-21.

32. Myers TJ, Khan T, Fazier O. Infectious Complications Associated with Ventricular Assist Systems. ASAIO J 2000;46:S28-36.

33. Kimball PM, Flattery M, Mcdougan F, et al. Cellular Immunity Impaired Among Patients on Left Ventricular Assist Device for 6 Months. Ann Thorac Surg 2008;85:1656-61.

34. Yamani MH, Chuang HH, Ozduran V, et al. The Impact of Hypogammaglobulinemia on Infection Outcome in Patients Undergoing Ventricular Assist Device Implantation. J Heart Lung Transplant 2006;25:820-4.

35. O'Horo JC, Saleh OMA, Stulak JM et al. Left Ventricular Assist Device Infections: A Systematic Review. ASAIO J 2018;64:287-94.

36. Leuck AM. Left ventricular assist device driveline infections: recent advances and future goals. J Thorac Dis 2015;7:2151-7.

37. Levy DT, Minamoto G. Role of Gallium SPECT-CT in the Diagnosis of Left Ventricular Assist Device Infections. ASAIO J 2015;61:e5-10.

38. Holman WL, Rayburn BK, Mcgiffin DC, et al. Infection in Ventricular Assist Devices : Prevention and Treatment. Ann Thorac Surg 2003;75:S48-57.

39. Sivaratnam K, Duggan J. Left Ventricular Assist Device Infections: Three Case Reports and a Review of the Literature. ASAIO J 2002;48:2-7.

40. Gordon SM, Schmitt SK, Jacobs M, et al. Nosocomial Bloodstream Infections in Patients With Implantable Left Ventricular Assist Devices. Ann Thorac Surg 2001;72:725-30.

41. Aslam S, Hernandez M, Thornby J, et al. Risk Factors and Outcomes of Fungal Ventricular- Assist Device Infections. Clin Infect Dis 2010;50:664-71.

42. Nienaber J, Wilhelm MP, Sohail MR. Current concepts in the diagnosis and management of left ventricular assist device infections. Expert Rev Anti Infect Ther 2013;11:201-10.

43. Hernandez GA, Breton JDN, Chaparro SV. Driveline Infection in Ventricular Assist Devices and Its Implication in the Present Era of Destination Therapy. Open J Cardiovasc Surg 2017;9:1179065217714216.

44. Thomas G, Hraiech S, Cassir N, et al. Venovenous extracorporeal membrane oxygenation devices - related colonisations and infections. Ann Intensive Care 2017;7:111.

45. Grasselli G, Scaravilli V, Di Bella S, et al. Nosocomial Infections During Extracorporeal Membrane Oxygenation: Incidence, Etiology, and Impact on Patients' Outcome. Crit Care Med 2017;45:1726-33.

46. Aubron C, Cheng AC, Pilcher D, et al. Infections Acquired by Adults Who Receive Extracorporeal Membrane Oxygenation Risk Factors and Outcome. Infect Control Hosp Epidemiol 2013;34:24-30.

47. O’Neill JM, Schutze GE, Heulitt MJ, et al. Nosocomial infections during extracorporeal membrane oxygenation. Intensive Care Med 2001;27:1247-53.

48. Sun HY, Ko WJ, Tsai PR, et al. Infections occurring during extracorporeal membrane oxygenation use in adult patients. J Thorac Cardiovasc Surg 2010;140:1125-1132.e2.

49. Hsu MS, Chiu KM, Huang YT, et al. Risk factors for nosocomial infection during extracorporeal membrane oxygenation. J Hosp Infect 2009;73:210-6.

50. Kim GS, Lee KS, Park CK, et al. Nosocomial Infection in Adult Patients Undergoing Veno-Arterial Extracorporeal Membrane Oxygenation. J Korean Med Sci 2017;32:593-8.

51. Haneke F, Schildhauer T, Schlebes AD, et al. Infections and Extracorporeal Membrane Oxygenation: Incidence, Therapy, and Outcome. ASAIO J 2016;62:80-6.

52. Buck ML. Pharmacokinetic changes during Extracorporeal Membrane Oxygenation: Implications for Drug Therapy of Neonates. Clin Pharmacokinet 2003;42:403-17.

53. Mulla H, Pooboni S. Population pharmacokinetics of vancomycin in patients receiving extracorporeal membrane oxygenation. Br J Clin Pharmacol 2005;60:265-75.

54. Shekar K, Fraser JF, Smith MT, et al: Pharmacokinetic changes in patients receiving extracorporeal membrane oxygenation. J Crit Care 2012;27:741.e9-18.

55. Mousavi S, Levcovich B, Mojtahedzadeh M. A systematic review on pharmacokinetic changes in critically ill patients : role of extracorporeal membrane oxygenation. 
Daru 2011;19:312-21.

56. Jamal JA, Economou CJP, Lipman J. Improving antibiotic dosing in special situations in the ICU: burns, renal replacement therapy and extracorporeal membrane oxygenation. Curr Opin Crit Care 2012;18:460-71.

57. Extracorporeal Life Support Organisation. ELSO ID TASK FORCE Recommendation Summary [internet], Ann Arbor, MI, USA 2012. Available online: https:// www.elso.org/Portals/0/Files/ELSO-ID-Task-Force-

Cite this article as: Tan Z, Zeng LA. Post-operative infection in mechanical circulatory support patients. Ann Transl Med 2020;8(13):831. doi: 10.21037/atm-20-1748
Recommendations-Summary.pdf

58. Paterson DL. "Collateral Damage" from Cephalosporin or Quinolone Antibiotic Therapy. Clin Infect Dis 2004;38:S341-5.

59. O’Horo JC, Cawcutt K. The Evidence Base for Prophylactic Antibiotics in Patients Receiving Extracorporeal Membrane Oxygenation. ASAIO J 2016;62:6-10. 